PROFESIONALES Y HERRAMIENTAS PARA EL DESARROLLO LOCAL Y SUS SINERGIAS TERRITORIALES. EVALUACIÓN Y PROPUESTAS DE FUTURO IX Coloquio Nacional de Desarrollo Local del GTDL-AGE 

ANTONIO MARTÍNEZ PUCHE, XAVIER AMAT MONTESINOS, ISABEL SANCHO CARBONELL y DANIEL SANCHIZ CASTAÑO (EDS.)

\section{PROFESIONALES Y HERRAMIENTAS PARA EL DESARROLLO LOCAL Y SUS SINERGIAS TERRITORIALES. EVALUACIÓN Y PROPUESTAS DE FUTURO}

IX Coloquio Nacional de Desarrollo Local del GTDL-AGE

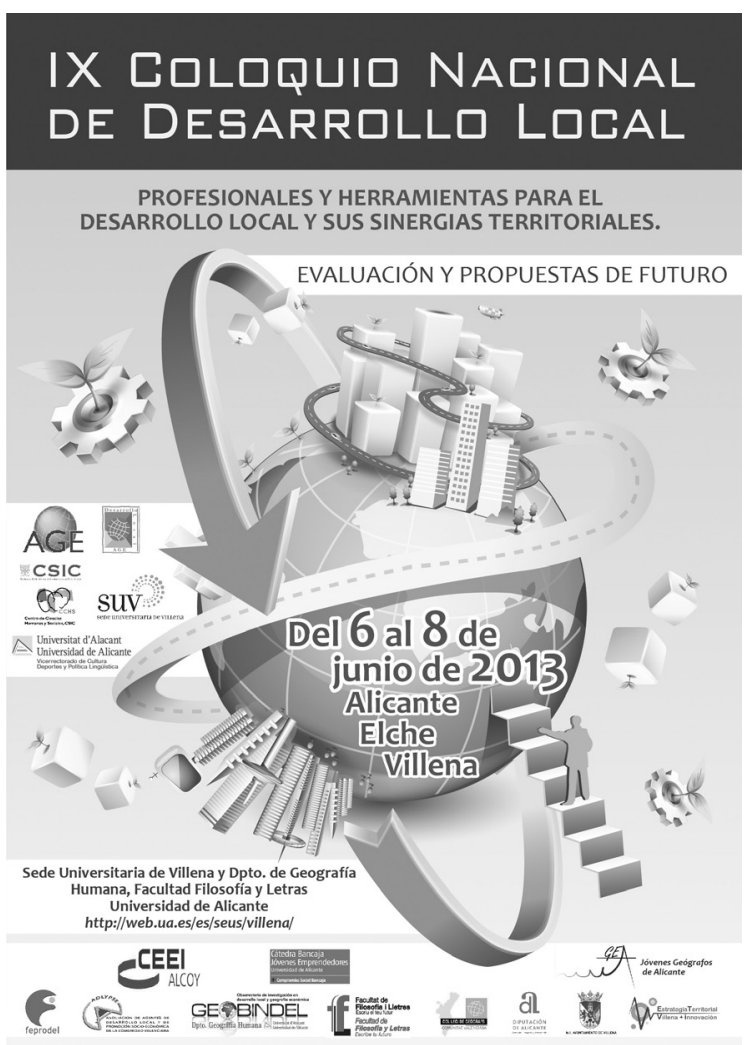


Este libro ha sido examinado y valorado por evaluadores ajenos a la Universidad de Alicante, con el fin de garantizar la calidad científica del mismo.

Publicacions de la Universitat d'Alacant

03690 Sant Vicent del Raspeig

Publicaciones@ua.es

http://publicaciones.ua.es

Telèfon: 965903480

(C) Antonio Martínez Puche, Xavier Amat Montesinos,

Isabel Sancho Carbonell y Daniel Sanchiz Castaño (eds.), 2016

(C) d'aquesta edició: Universitat d'Alacant

ISBN: 978-84-16724-00-0

Dipòsit legal: A 92-2016

Disseny de coberta: candela ink

Composició: Página Maestra (Miguel Ángel Sánchez Hernández)

Impressió i enquadernació: Guada Impresores

\section{unte \\ Unión de Editoriales
Universitarias Españolas \\ WWW.une.es
WWA}

Esta editorial es miembro de la UNE, cosa que garantiza la difusión y comercialización nacional y internacional de sus publicaciones.

Reservados todos los derechos. Cualquier forma de reproducción, distribución, comunicación pública o transformación de esta obra sólo puede ser realizada con la autorización de sus titulares, salvo excepción prevista por la ley. Diríjase a CEDRO (Centro Español de Derechos Repográficos, www.cedro.org) si necesita fotocopias o escanear algún fragmento de esta obra. 


\title{
LOS PROCESOS DE INNOVACIÓN SOCIAL MEDIANTE LA PARTICIPACIÓN CIUDADANA COMO ESTRATEGIA PARA EL DESARROLLO LOCAL $^{1}$
}

\author{
Dr. Vicente Manuel Zapata Hernández \\ Profesor Titular de Geografía Humana \\ Universidad de La Laguna \\ vzapata@ull.es
}

"Las relaciones a través del diálogo nos permitirán encontrar las soluciones"

(Marco Marchioni, 2013)

RESUMEN

La ciudadanía necesita nuevos cauces de participación, que deben ir de la mano de renovados procedimientos y de la complicidad directa de los políticos. También apostando por una participación, no tanto representativa y orgánica, sino voluntaria y legitimada por toda la ciudadanía. En esta ponencia se presentan estudios de caso en Canarias y en la Comunidad Valenciana, que aportan un renovado enfoque aplicado sobre procesos y procedimientos, que sin duda enriquecen instrumentos para favorecer un desarrollo local inclusivo e integrado.

Palabras Clave: Desarrollo local, participación ciudadana, gobernanza, innovación territorial, foro económico y social.

\section{THE PROCESSES OF SOCIAL INNOVATION BY MEANS OF THE CIVIC PARTICIPATION AS STRATEGY FOR THE LOCAL DEVELOPMENT}

1 La exposición de la ponencia se realizó de manera conjunta con integrantes del Grupo de Trabajo del Foro Económico y Social de Villena, experiencia que forma parte asimismo del texto, al considerarse original e innovador su proceso participativo de conformación entre los años 2012 y 2014 (foto 9 y 10). 


\section{ABSTRACT}

The citizenship needs new participation beds that they should go of the hand of having renovated procedures and of the direct complicity of the politicians. Also betting on a participation, not so much representative and organic, but voluntary and legitimated by the whole citizenship. In this report they show up case studies in Canaries and in the Valencian Community that you/they contribute a renovated focus applied on processes and procedures that without a doubt enrich instruments and integrated development.

Key words: Development local, civic participation, government, territorial innovation, economic and social forum.

\section{INTRODUCCIÓN}

La ciudadanía es cada vez más consciente de que el afianzamiento de los derechos sociales está estrechamente relacionado con su grado de participación en la vida pública, recuperando los referentes colectivos y la idea-sentimiento de comunidad, asumiendo que es posible realizar contribuciones esenciales a la mejora de la realidad compartida, superando el estricto esquema de delegación que supone la Democracia Representativa. Ciudadanía y participación social están vinculadas, por lo tanto, entendida la segunda como una herramienta que nos permite intervenir de manera individual, grupal y colectiva en nuestro entorno, conjugando la aportación de sus distintos protagonistas, para avanzar en la definición de proyectos coherentes y viables de territorio; convertidos en procesos de innovación social, suponen uno de los fundamentos del desarrollo en el marco local.

Y es que, uno de los permanentes desafíos de la participación social es que sirva a los fines del desarrollo de los territorios, convirtiendo a la comunidad en protagonista de su destino, con la aspiración de formular su estrategia particular, fruto de un análisis compartido de la realidad local que sirva para identificar las principales dificultades y oportunidades de progreso. Proyecto comunitario, sinónimo de estrategia colectiva en cuya definición participen todos los agentes que interactúan en el territorio de referencia, considerando además un enfoque intergeneracional que propicie la obtención de resultados a la medida de todos y de todas, y que, además, enganche a la comunidad en su puesta en marcha y periódica revisión.

Proyecto comunitario que ayudará a orientar de forma conveniente el proceso de desarrollo en cada momento, aprovechando las capacidades, la experiencia y los recursos propios. La definición de dicho proyecto o estrategia supone el planteamiento de una dinámica de trabajo que contribuirá a recuperar la ilusión de muchas personas, grupos, colectivos y comunidades, en relación con la mejora de su entorno vital, siendo conscientes de que su participación es 
clave para avanzar si se logra conformar una mínima organización que integre de manera adecuada todas las aportaciones. Ese es uno de los retos que todavía tienen pendiente la mayor parte de territorios locales, para el que, con frecuencia, se dispone del mejor capital humano, así como de recursos e instrumentos junto a iniciativas que ya han dado sus frutos y pueden constituir válidas referencias, pero que no se han sabido articular adecuadamente.

La organización creativa de las comunidades locales es entonces clave para encontrar renovadas formas de enfrentar una realidad cada vez más compleja en la escala en que se desenvuelven cotidianamente las personas, conjugando los distintos puntos de vista para conseguir elementos de cohesión. Realidad que plantea viejos y modernos problemas, identificados conjuntamente, a partir de un diagnóstico que propicie el desarrollo de marcos de actuación coherentes que contengan propuestas viables optimizando los recursos disponibles o movilizando nuevos. En este contexto, la conformación de redes estables para el fomento de la participación social mediante la acción compartida, ofrece una cierta garantía para la sostenibilidad de los procesos que tienen como objetivo esencial mejorar las condiciones de vida de la población, partiendo de sus propias necesidades, posibilidades e intereses.

En este marco de análisis, se pretende ahondar en la reflexión sobre el papel que desempeñan los procesos de innovación social como palanca para el desarrollo local, valiéndose de la participación de la ciudadanía como instrumento que favorece la generación de contextos propicios para que la creatividad comunitaria se plasme en proyectos y estrategias que repercutan en la mejora colectiva. Se parte de la premisa de que, sólo desde la concurrencia y adecuada organización de los diversos actores que protagonizan cada realidad, se generan procesos de desarrollo con posibilidad de propiciar las transformaciones necesarias que procuren el bien común, centrándose en los intereses generales, para lo que, además, deben ser sostenibles en el tiempo adaptándose a las condiciones y aprovechando las oportunidades de cada momento.

El trabajo se articula de este modo en tres apartados, siendo el primero el que aborda los modernos retos que debe afrontar la participación social considerando sus distintos protagonistas. El segundo trata el significado de aquélla en los procesos de desarrollo en el marco local, enfatizando las más recientes perspectivas y recomendaciones europeas. Concluye el tercero con el relato de una experiencia ilustrativa, actualmente vigente en el municipio de Villena de la Comunidad Valenciana, concebida desde y plasmada en un contexto particular conjugando distintas aportaciones mediante un objetivo común. El epígrafe final formula diversos desafíos que siguen planteándose a la participación social desde la mirada del desarrollo local, como corolario de la reflexión realizada a modo de ensayo. 


\section{LOS MODERNOS RETOS DE LA PARTICIPACIÓN SOCIAL MIRANDO AL TERRITORIO ${ }^{2}$}

Los procesos participativos se basan en la conformación colectiva de contextos, sistemas y estructuras que promuevan las relaciones mediante el diálogo entre los protagonistas de cada realidad, siendo el territorio el ámbito fundamental para establecer dichos vínculos, al margen de que también podamos favorecerlas en las organizaciones a todos los niveles. Allí es donde podemos construir referencias comunes fruto de la aportación de todos y de todas. Primero en los territorios próximos, en la escala local, para más tarde ampliar la mirada hasta conseguir nuevas integraciones funcionales, puesto que, si no damos respuesta a lo local desde lo local, posiblemente será imposible producir cambios más amplios que repercutan asimismo en el bienestar general.

De este modo, en cualquier iniciativa para promover la participación activa de la ciudadanía habría que considerar ineludiblemente la importancia del enfoque local, la perspectiva ascendente, la mirada de abajo hacia arriba. Y eso supondría establecer mecanismos para que en su formulación participen todos los agentes y sectores sociales que lo deseen, e incluso, la ciudadanía a título individual. Y también todos los territorios, con la intención de generar complicidades y redes que aseguren la permanencia de procesos que aspiren a la mejora continua y logres trascender a otras escalas geográficas.

Y es que, los territorios necesitan dotarse de una visión estratégica, construida sobre todo en función de una serie de principios y valores enunciados por la comunidad de forma participativa. Llegamos ahí a través de procesos dinámicos adaptados a cada situación, motivando a las personas y sacando a relucir el talento de la gente, sobre todo a partir de la utilización de metodologías originales aplicadas de manera planificada sobre los elementos que configuran el potencial de desarrollo local. Esto genera una forma particular de hacer las cosas y deriva en la concreción de modelos de vida y de desarrollo que envuelven todas las acciones y producciones humanas, y que, además, suponen nuestra mejor tarjeta de presentación y proyección exterior.

La participación ciudadana debe conducir además hacia la construcción de una sociedad cada vez más consciente, comprometida y solidaria, corresponsable con el buen gobierno que aspire a gestionar de modo adecuado los temas comunes. Gestión eficiente, inclusiva y transparente, que cuente con canales siempre abiertos para favorecer la concurrencia de los distintos actores en cada contexto territorial. Gobierno abierto que asimismo enfatice el acceso a la información disponible, para que podamos definir los mejores proyectos y tomar las decisiones más pertinentes. Para ello, será preciso prestar atención a la ne-

2 Este apartado se basa en los documentos elaborados por el autor para el proceso de formulación del bloque de 'Participación Ciudadana' del Pacto por Tenerife para Canarias, desarrollado durante los años 2013 a 2015 fruto del impulso de la Universidad de La Laguna a partir de la iniciativa de las principales fuerzas políticas regionales. 
cesaria renovación de los marcos administrativos y legislativos, que permitan incorporar cambios que propicien el debate colectivo y la consideración de las iniciativas surgidas de la comunidad en cada momento.

Recuperando la reflexión anterior acerca de la necesaria creación de referencias comunes, parece imprescindible en la situación actual que volvamos a encontrarnos en el mundo real, juntándonos creativamente, saliendo de nuestros ayuntamientos, empresas, aulas, despachos profesionales, e incluso, domicilios, en los que nos hemos bunkerizado abandonando planteamientos más comunitarios para desarrollar integraciones corporativistas y comportamientos individualistas. Es preciso entonces reconstruir el diálogo, la confianza y la acción compartida entre los que gobiernan y gestionan y los que son gobernados y gestionados, entre los administradores y los administrados. Y esto nos implica a todos y a todas; no sólo es una tarea para los responsables públicos, se trata de un reto colectivo que tiene su expresión más factible en el marco local.

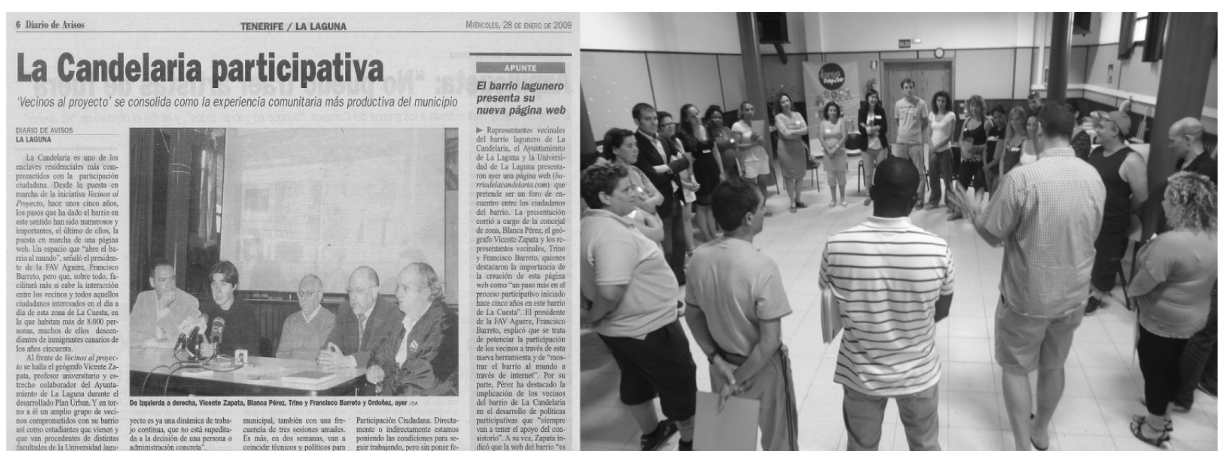

Fotos 1 y 2. Visibilidad y trabajo comunitario en barrios de Tenerife.

Fuente: Vicente Zapata

La apuesta por la participación de la ciudadanía en los asuntos públicos desde la Administración, tanto en su dimensión política como técnica, implica, en primer lugar, que ésta pierda sus múltiples incertidumbres y miedos hacia los procesos participativos y sus efectos; en segundo lugar, que favorezca el desarrollo de estructuras e instrumentos que permitan la confluencia de actores en torno a cuestiones de interés común; y en tercer lugar, que encuentre fórmulas para que la ciudadanía participe más en el día a día de la propia Administración en sus diferentes niveles. También es fundamental que coloque adecuadamente eso que se denomina 'Participación Ciudadana' en el organigrama institucional, por ejemplo, en el caso de los municipios, reconsiderando la propia existencia de áreas de gobierno específicas en la materia y situándola como eje transversal de la acción pública, hacia adentro y hacia afuera. 
Revisando y adaptando asimismo la reglamentación en materia de participación ciudadana a los nuevos tiempos, fruto de una labor concertada que considere la pluralidad existente; y racionalizando y haciendo más operativos y útiles los órganos consultivos, incluso confiriéndoles cierta capacidad de decisión. Algunas de las líneas estratégicas de actuación de la Administración deben ser tan simples como la de invertir en educación para la participación y la de favorecer el intercambio de buenas prácticas con efecto demostrativo en materia de participación social, porque las hay, pero tienen todavía insuficiente visibilidad y débil proyección. La participación ciudadana no debe ser un elemento decorativo y propagandístico en las instituciones, sino un potente instrumento para el cambio del que se beneficie la comunidad en su conjunto.

También toca de lleno este tema a los que intervienen desde el plano científico, profesional y técnico, cambiando su forma de hacer y abriéndose de manera comprometida a una labor más colaborativa e incluso interdisciplinar. Suprimiendo barreras anacrónicas e inútiles y propiciando el encuentro con el resto de protagonistas de la realidad. Su labor puede ser fundamental en la conjunción del triángulo de actores, pero para ello deben incorporar nuevos conceptos y métodos de trabajo que favorezcan la interacción, incluso modulando su lenguaje para que la relación con la ciudadanía pueda ser mucho más fluida y positiva, en base a una comunicación que aspire siempre a ser bidireccional y más cercana, con todo lo que esto implica. Existen múltiples oportunidades en la esfera de la motivación, la facilitación, la mediación, el acompañamiento, la sistematización, la difusión de los procesos participativos, y por lo tanto, de la gobernanza territorial. Es posible realizar importantes aportaciones desde esta esfera en el cómo se deben hacer las cosas.

Y la ciudadanía, aunque todos y todas somos ciudadanía, adquiere un enorme protagonismo en la nueva situación. Particularmente nos referimos al papel que desempeñamos como vecinos y vecinas de un territorio, reivindicando nuestro derecho a participar plenamente en la vida pública. Para comenzar, recuperando los referentes colectivos y la idea de comunidad, asumiendo que es posible realizar contribuciones fundamentales a la mejora de la realidad compartida, superando el estricto esquema de la delegación que supone la Democracia Representativa. Un logro esencial, pero no suficiente en el contexto actual y futuro; y en esto nos debemos empeñar, revisando en primer lugar el modelo de vida que protagonizamos y el rol que en él desempeña nuestra participación en los asuntos colectivos.

A partir de aquí, es preciso analizar de forma crítica y con vocación de renovación el funcionamiento de las estructuras organizativas, que tanto desde el punto de vista territorial como sectorial seguimos manteniendo para estimular y canalizar la participación ciudadana, muchas de las cuales ya no responden a las nuevas necesidades, inquietudes y formas de relación social; porque la sociedad se ha transformado enormemente en las últimas décadas y en muchos 
casos esto no se ha visto reflejado en la evolución de sus organizaciones representativas. Y mucho menos en su adaptación a los modernos retos de la gobernanza territorial, que implica una relación más dinámica con los otros actores. Por lo tanto, miremos hacia adentro antes de pretender cambiar lo de afuera.

Cuadro 1. Retos de la participación social con incidencia en los procesos de desarrollo local

- Re-configuración del tejido asociativo y renovación de sus formas de actuación

- Formalización de alianzas y establecimiento de redes consistentes, estables y duraderas

- Emergencia de nuevos líderes sociales no comprometidos políticamente

- Inclusión de personas autónomas 'no afiliadas' que desean participar de modo activo

- Aplicación de renovadas metodologías que fomenten la acción grupal y comunitaria

- Desarrollo de marcos de participación más dinámicos, operativos y gratificantes

- Generalización de una actitud más inclusiva y profundización en la perspectiva intercultural

- Incorporación de los avances tecnológicos en el desarrollo de procesos participativos

- Combinación de las dimensiones sectorial y territorial en la perspectiva del desarrollo

- Superación de inútiles corporativismos para desarrollar planteamientos más integrales

- Sostenimiento de los procesos participativos más allá de los periodos legislativos

- Mayor permeabilidad de las iniciativas de desarrollo a la participación social en todas sus fases

Fuente: Elaboración propia.

Entre las posibilidades de todos también está reconocer e incluso premiar las experiencias participativas que signifiquen un ejemplo para el conjunto de la sociedad, de modo que se vaya produciendo un cambio cultural y de valores que reoriente la mirada hacia lo común, que enfatice los referentes generales frente a los intereses particulares y corporativos, que rescate la importancia de lo comunitario ${ }^{3}$. Ejercicio prioritario en relación con lo que deberíamos

3 Reflexiones basadas en el amplio legado de Marco Marchioni en relación con la recuperación de los intereses generales y el enfoque comunitario del desarrollo. 
proyectar hacia las personas que tendrán que ir asumiendo progresivamente liderazgos en todos los ámbitos de nuestra realidad, que protagonizarán nuestro futuro inmediato. También cabe valorar las conductas más comprometidas de la población a título particular, porque cada vez es más necesario favorecer la concurrencia de la ciudadanía no organizada, de las personas que de manera autónoma desean contribuir al bien común y que no encuentran adecuados cauces para su expresión y participación en las dinámicas sociales que promueven las organizaciones tradicionales.

De este modo, los distintos actores deben aspirar a establecer los vínculos necesarios que deriven en un compromiso de acción conjunta, encontrando espacios y momentos para el encuentro creativo que permita conformar estrategias con las que enfrentar los retos que trae cada día la realidad que se comparte. Cualquiera de ellos puede dar el primer paso -debe dar el primer paso-, siempre que su objetivo sea lograr la mejor inclusión posible del resto en el marco en que se interactúa. Cada territorio debe configurar además su modelo relacional desde el conocimiento y la experiencia adquiridos, pero también desde la identificación de sus particularidades, necesidades y fines, siendo asimismo consciente de sus recursos y de sus posibilidades, haciendo un esfuerzo permanente para la mejora de sus capacidades.

La preparación para la participación a todos los niveles será entonces una tarea esencial, considerando que supone un instrumento que ofrece su mejor versión y más adecuado rendimiento en los tiempos medios y largos, por lo que habrá que superar su frecuente encorsetamiento en esquemas que responden más a la dinámica político-partidista que al pulso social. Sólo así será una herramienta plenamente compatible con los fines del desarrollo local en clave de proceso, esencial para lograr aglutinar al conjunto de actores alrededor de un proyecto compartido y enunciado desde la convicción de que cada persona puede ser sujeto del cambio, pero que sólo la comunidad será impulsora y protagonista de su consolidación y difusión.

\section{La PARTICIPACIÓN SOCIAL COMO PILAR DEL DESARROLlO LOCAL}

La participación social debe constituir un componente esencial de la estrategia de desarrollo de cualquier ámbito geográfico, como ya se ha destacado. Su expresión a través de cauces adecuados redunda en la configuración de un proceso mediante el que las personas, los colectivos y la comunidad adquieren más responsabilidad real en el gobierno de las cuestiones compartidas; aumentan asimismo su capacidad y disposición a ser solidarios y cooperativos, elevan su nivel de información y conciencia sobre los asuntos públicos y la importancia de alcanzar y ejercitar opiniones bien fundamentadas, y además, aprenden a considerar y entender las razones de los otros actores. Por ello, es preciso favorecer la gestión participativa de los procesos de desarrollo local, facilitando 
la concurrencia de la ciudadanía mediante la habilitación de canales eficaces y utilizando modernos métodos de trabajo colaborativo.

Desde una perspectiva operativa, la participación social permite conocer de manera directa las necesidades, los intereses y las aspiraciones de la población, enriqueciendo las iniciativas que se promueven a través de la canalización de múltiples aportaciones. Asimismo propicia ahondar en la información, sensibilización y responsabilidad de la ciudadanía, establecer redes que propician el trabajo común, fomentar la creación de modernos espacios de encuentro y la constitución de nuevos colectivos, asegurar la viabilidad y el éxito de los proyectos emprendidos de forma compartida, y además, encauzar adecuadamente los procesos de desarrollo en los territorios asegurando su sostenibilidad. De ahí, que desde el gobierno local sea fundamental fortalecer los valores democráticos para permitir una mayor concurrencia social en la dinámica de toma de decisiones.

Sin embargo, el reto de la dinamización de los territorios y de la participación activa de la ciudadanía choca, casi siempre, con dos problemas fundamentales: la falta de compromiso e implicación de la sociedad civil en la

\section{Cuadro 2. Cuestiones clave de la participación social en el desarrollo local}

- La ciudadanía debe ser cada vez más consciente de su realidad y de la capacidad que posee para influir en ella

- La colaboración de los actores en el marco local supone la base para afianzar el proceso de desarrollo compartido

- Los procesos participativos surgen y se orientan a partir del diagnóstico permanentemente actualizado de la realidad local

- Las comunidades disponen de recursos endógenos que les permiten afianzar y singularizar su estrategia de desarrollo

- Las iniciativas comunitarias deben adquirir la forma de proyecto para superar la dispersión de esfuerzos

- La planificación es básica para orientar adecuadamente los procesos de desarrollo local de base comunitaria

- El trabajo comunitario debe implicar a los colectivos sociales potenciando su compromiso con el proceso de desarrollo local

- La visión compartida y a largo plazo de la comunidad convierte sus iniciativas en procesos de mayor alcance y trascendencia

- Las redes son imprescindibles para que la ciudadanía sea protagonista de su realidad

- La autoestima es una de las fuentes esenciales del progreso comunitario

Fuente: Claves para el desarrollo del trabajo comunitario en nuestros barrios, Vecinos al Proyecto, 2010. 
construcción de un proyecto de vida común en el territorio; y la obsolescencia de las estructuras y de los instrumentos que se utilizan para promover y afianzar la participación social. Por eso, es preciso idear y articular modernas estrategias para redefinir las bases y los métodos que sirven para canalizar el interés de los habitantes hacia su proceso de desarrollo, y además, para que se proyecte sobre planteamientos sólidos, que vayan más allá de la realización de meras iniciativas puntuales con escasa repercusión en las diferentes dimensiones de la realidad de cada lugar.

Pese a las dificultades expresadas, las actuales tendencias y casos de éxito reafirman la convicción de que se debe avanzar en la profundización de los planteamientos originales del desarrollo local de base comunitaria, que han sido desvirtuados en muchos casos y se ha concluido en la expresión de procesos burocratizados y dirigidos sobre todo por la Administración. Aquellas premisas sitúan el acento en la confluencia de actores que marcan retos y formulan objetivos de manera compartida, y además, definen e implementan estrategias de desarrollo con enfoque flexible, integral y multisectorial, basadas en la optimización del potencial endógeno y poniendo en valor las propias capacidades de las comunidades locales.
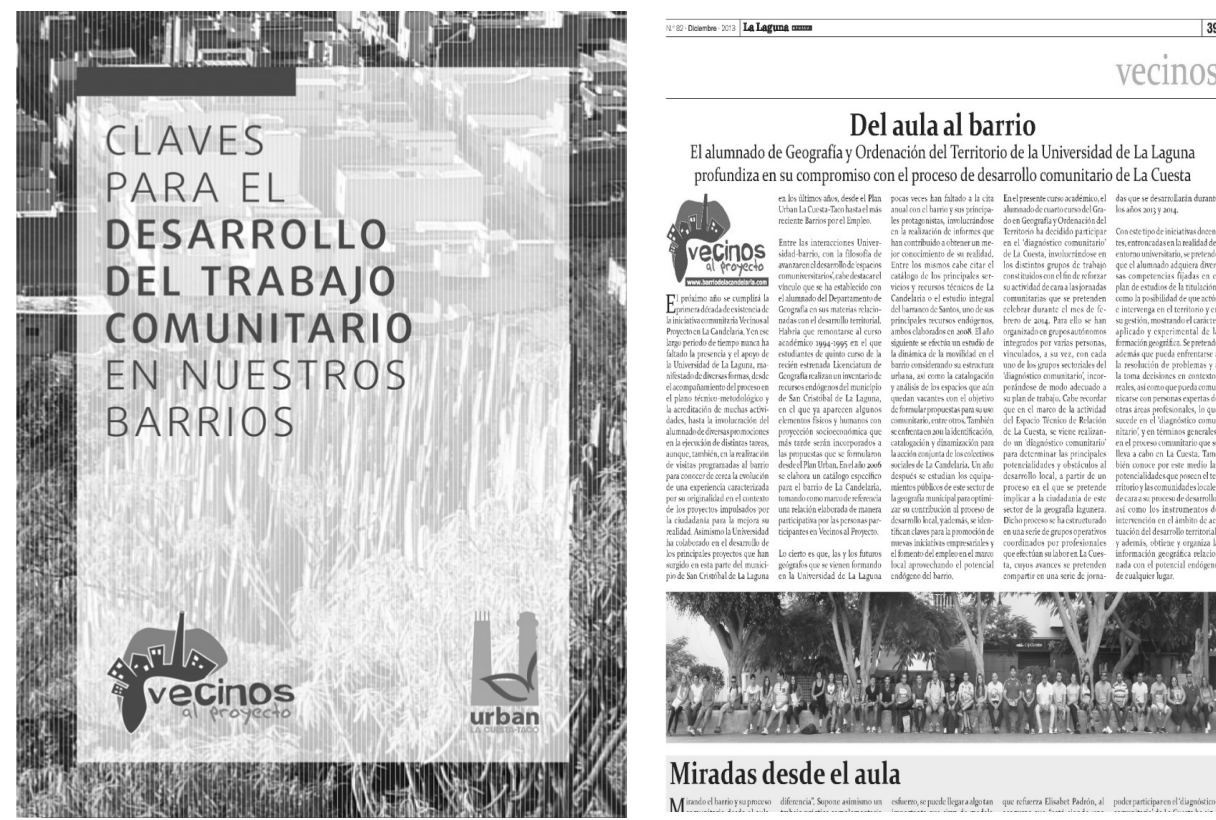

Fotos 3 y 4. Guía de trabajo comunitario de Vecinos al proyecto y acciones desarrolladas por los alumnos de Geografía y Ordenación del Territorio de la ULL 
Se trata de recuperar el enfoque ascendente (bottom-up) y el principio de subsidiariedad en la escala local, planteando respuestas propias a los problemas y retos que ofrece la realidad en cada momento, donde una ciudadanía cada vez más consciente, activa y protagonista, se compromete con su proceso de desarrollo y se implica en la gobernanza del territorio. Esta dinámica se articula en torno a grupos de acción, plenamente autónomos y democráticos, que elaboran de forma colaborativa estrategias integradas de desarrollo favoreciendo procesos participativos altamente inclusivos, en los que la Administración puede seguir desempeñando un papel activo y además esencial, pero no capitalizando todo el protagonismo.

En este sentido, la propia Unión Europea en sus más recientes manifestaciones es crítica con el modelo descendente (top-down), basado en la fórmula del gasto dirigido desde arriba, que habitualmente está acompañado de marcos de gestión rígidos o impuestos y que no logra estimular ni entroncar con la ciudadanía, apostando por lo que denomina desarrollo local participativo ${ }^{4}$. Por ese motivo desea reforzar la difusión de las enseñanzas de iniciativas comunitarias con alto carácter innovador, responsable y participativo, como han sido LEADER (Fondos Estructurales), URBAN (FEDER) o EQUAL (FSE). Pretende de este modo avanzar hacia la consecución de los objetivos de la Estrategia Europa 2020 de crecimiento inteligente, sostenible e integrador, fomentar la cohesión territorial y lograr los fines específicos de sus políticas ${ }^{5}$.

El desarrollo local participativo tiene un importante valor añadido, según el Comité de las Regiones de la Unión Europea, porque permite avanzar en la participación, la concertación y la cooperación de la población y del conjunto de actores públicos y privados que se desenvuelven en el marco local. También favorece la adecuación de cada estrategia de desarrollo a las necesidades particulares de cada territorio, promoviendo una poderosa influencia en la colaboración multinivel e intersectorial, la movilización del capital humano, la

4 Dictamen del Comité de las Regiones (2013/C 17/05 - DO 19.01.13). En sus recomendaciones políticas, el Comité de las Regiones "destaca que el desarrollo de las capacidades es una característica fundamental del Desarrollo Local Participativo y considera que se deben poner a disposición los recursos suficientes para que los agentes locales pueda preparar y llevar a la práctica sus estrategias locales" (punto 10 del Documento de Referencia cuyo ponente es Graham Garvie, DO C 17/19, 19.01.13).

5 La Comisión Europea asimismo hace énfasis en el concepto de desarrollo a cargo de las comunidades locales, incluido en la política de cohesión 2014-2020 mediante un apoyo más explícito en forma de marco jurídico común y reglas armonizadas en los cincos fondos del Marco Estratégico Común. En este sentido, señala en sus documentos oficiales que "aumentará la coherencia y fomentará la creación de estrategias locales financiadas por varios fondos a cargo de la comunidad local". Su propuesta favorecerá los territorios subregionales específicos por medio de grupos de acción local, promoviendo la definición de estrategias de desarrollo local por áreas integradas y multisectoriales que deben considerar las necesidades y potencialidades locales, poniendo en valor sus características innovadoras, la creación de redes y la cooperación. 
capacidad de innovación y el enfoque integrado, entre otros aspectos relevantes, que van en la línea de conceder más protagonismo a las premisas del enfoque del Gobierno Abierto.
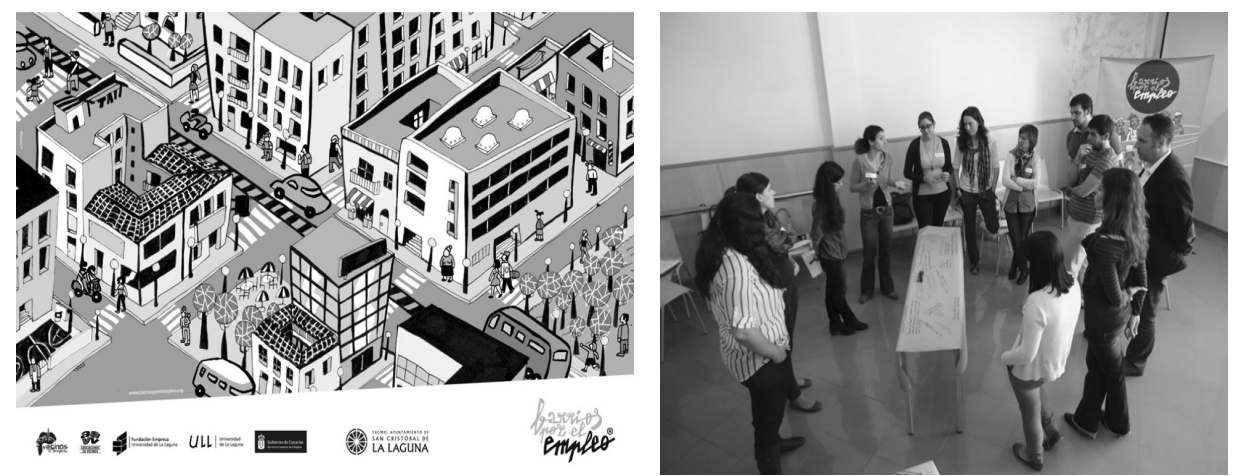

Fotos 5 y 6. Publicidad y trabajo participativo en "Barrios por el empleo"

Fuente: Vicente Zapata

Para enfrentar los retos y alcanzar los objetivos expuestos se sugiere reforzar la asistencia técnica en el marco local, buscando nuevas fórmulas más flexibles y menos burocratizadas para la dinamización económica y social del territorio, en las que la ciudadanía tenga un renovado protagonismo junto a los equipos de trabajo, imprescindible para alcanzar los fines establecidos de manera conjunta. Se pretende que las acciones impulsadas adquieran amplia repercusión comunitaria y se valora también su dimensión cualitativa, ahondando en el enfoque ascendente y resaltando la idea de proceso, esto es, en las construcciones de abajo hacia arriba ${ }^{6}$.

En este sentido, el Comité de las Regiones estima que el desarrollo local participativo es un instrumento fundamental para el desarrollo armonioso de las regiones, tanto urbanas como rurales, y que, además, puede facilitar e impulsar la recuperación económica y la creación de empleo, aspectos clave en la coyuntura actual. Se expresa con más posibilidades en territorios en los que existe masa crítica suficiente, que permita definir e implementar de manera efectiva una estrategia local de desarrollo integrada, a partir de la configuración colectiva de objetivos comunes. Su consolidación pasa por incidir en la motivación de la ciudadanía, facilitando recursos para su organización y la adquisición de competencias necesarias para liderar este tipo de procesos, alentando asimismo la involucración de la Administración en sus diferentes niveles.

6 Se trata de planteamientos que coinciden con los que ha venido desarrollando el proyecto Intervención Comunitaria Intercultural que promueve la Obra Social "la Caixa" desde 2010 y se ha extendido a cuarenta territorios repartidos por la geografía española. 
Cuadro 3. Orientaciones para la acción técnica en un proceso de desarrollo de base comunitaria

- Enmarcar cada proceso en un territorio bien delimitado: barrio o pueblo (espacio coherente)

- Establecer un clima de confianza entre los actores: continuidad y responsabilidad

- Organizar una amplia y estable red de personas y entidades involucradas

- Propiciar el protagonismo a los actores locales con todas sus consecuencias

- Flexibilizar y adaptar los instrumentos metodológicos utilizados a cada realidad

- Fundamentar la acción en la implicación efectiva de los/as participantes

- Desarrollar canales para encajar de modo adecuado los elementos exógenos (aportación exterior)

- Construir un sistema o estructura con creciente autonomía, interna y externa

- Generar un clima de confianza hacia las instituciones públicas

- Considerar y ser conscientes de los diferentes ritmos del proceso

- Combinar lo macro y lo micro, las estrategias generales y los asuntos cotidianos

- Registrar la dinámica del proceso con todos sus detalles (caja negra)

- Habilitar, visibilizar y facilitar los espacios de trabajo operativos del proyecto-proceso

- Formalizar el proyecto-proceso para fortalecer su autonomía funcional

- Considerar siempre que todos/as podemos aportar algo para que el proceso avance

Fuente: Elaboración propia.

En todo caso, considerando nuestra propia experiencia, para que se pueda iniciar con garantías un proceso de desarrollo local de base comunitaria es preciso tener presente una serie de aspectos estrechamente interrelacionados entre sí. En primer lugar, la delimitación y justificación del área de intervención, puesto que es fundamental abarcar un territorio coherente, en la medida en que su escala sea asumible y se reconozcan en él los actores locales. La identificación de estos y de sus relaciones funcionales, reconociendo sus conflictos y destacando sus potencialidades, supone asimismo un ejercicio esencial, dado que, sobre los vínculos de distinto tipo se construyen los proyectos colectivos. También se requiere explorar la estructura técnica existente, particularmente sus capacidades y limitaciones, así como realizar una adecuada cartografía de 
las redes disponibles y potenciales, internas y externas al sistema en que se va a intervenir.

La valoración de la experiencia colectiva acumulada en materia de desarrollo propicia asimismo que se pueda partir de lo existente, enfatizando aportaciones y esfuerzos anteriores, que siempre van a permitir sumar adhesiones. A partir de aquí, parece esencial tomar conciencia de la realidad compartida a través del diagnóstico comunitario, y en paralelo, afrontar el reconocimiento y análisis del potencial-endógeno, esto es, de los factores estratégicos que se encuentran en el espacio de referencia para alentar un proceso de desarrollo territorial. Ambas líneas de trabajo permitirán avanzar hacia la formalización de una estrategia compartida, y por lo tanto, contribuirán a reforzar la idea de espacio-proyecto, esto es, de que cada territorio cuente con su propio plan.

\section{CREATIVIDAd COMUNitaria PARA La INNOVACIÓN SOCIAL: VilLENA Y SU FORO}

La creatividad comunitaria se entiende como la capacidad de la sociedad para promover cambios que deriven en la mejora de sus condiciones de vida, a partir de la generación de nuevas ideas o conceptos -y también, de nuevas asociaciones entre ideas y conceptos ya conocidos- que producen respuestas originales a los retos que se deben enfrentar de forma conjunta. Nos remite a la innovación, a la inventiva, al descubrimiento, a la originalidad, a la espontaneidad, al pensamiento creativo, al trabajo colaborativo, a la flexibilidad, a la generación de conocimiento, a la gestión del conflicto, al encuentro de soluciones compartidas para la transformación positiva de la realidad. Las comunidades deben ser conscientes de que disponen de dicho potencial y de que puede ponerse al servicio de su proceso de desarrollo, mediante la definición de estrategias que integren a los actores que se desenvuelven en el contexto local ${ }^{8}$.

Este tipo de capacidades debe ser fomentada y cultivada a partir del encuentro ciudadano, creando espacios y generando ambientes que motiven el trabajo colaborativo -idear conjuntamente- en el marco de la vida cotidiana, que repercuta en el planteamiento de iniciativas que contribuyan a optimizar los recursos propios. Por lo tanto, creatividad comunitaria y desarrollo local constituyen conceptos interdependientes: la primera incide en el segundo si se estimulan y canalizan adecuadamente las capacidades creativas de la sociedad; y el segundo se apoya en la primera si se logra abrir el proceso de desarrollo a la participación social. La educación y la formación para la creatividad y el

7 Proceso participativo para la constitución del Foro Económico y Social de Villena en el marco de actuación de la Estrategia Territorial de Villena más Innovación (ETV+i).

8 Reflexiones sobre creatividad comunitaria obtenidas junto a las personas participantes en el proceso formativo 'creatividad comunitaria para el desarrollo territorial', realizado en la Universidad de La Laguna en el marco del Plan Universitario de Empleo entre los años 2009 y 2010 . 
desarrollo, suponen elementos clave para que la ciudadanía sea consciente, propicie y participe de manera compartida de esa fructífera relación.

Los procesos de innovación social tienen mucho que ver con el estímulo de la creatividad comunitaria, como ya se ha dicho, cuajando sobre todo allí donde se desarrollan las condiciones para que la ciudadanía se comprometa realmente con determinadas iniciativas que suponen elementos de cambio y poder transformador de la realidad compartida. Una de estas experiencias se viene produciendo en el municipio alicantino de Villena, en el marco de actuación de la Estrategia Territorial de Villena más Innovación (ETV+i) ${ }^{9}$, que comenzó a desarrollarse en abril de 2012 como proyecto financiado por el Ayuntamiento de Villena y coordinado por el Departamento de Geografía Humana de la Universidad de Alicante.

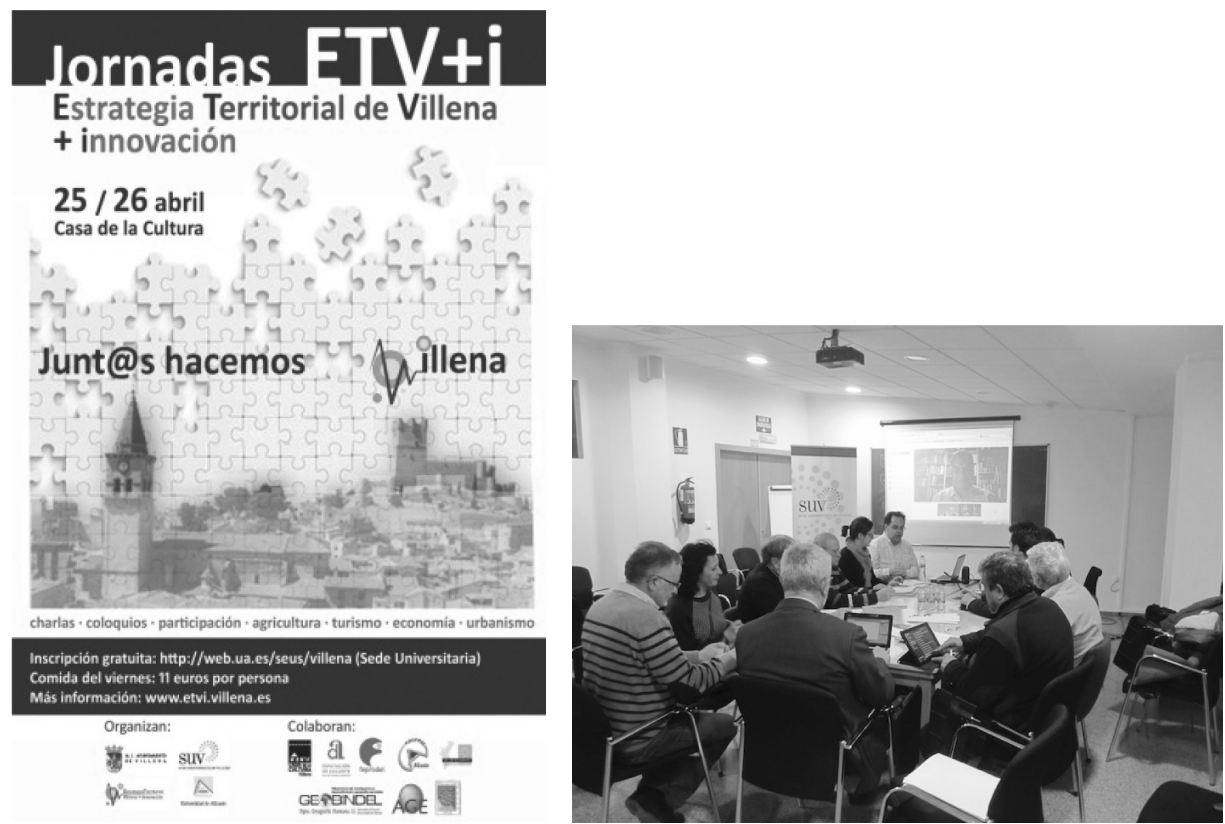

Foto 7 y 8. Jornadas de divulgación y trabajo de la ETV+i e imagen del grupo promotor del Foro Económico y Social en la sede universitaria de Villena (UA)

Fuente: Salvador Martínez Puche

A lo largo del proceso de trabajo general se han realizado múltiples análisis, junto al desarrollo de talleres, jornadas, coloquios y asambleas ciudadanas, dinamizados por el equipo de la ETV+i. Durante esta intensa labor se procuró favorecer la reflexión colectiva y compartida en torno al modelo territorial y a la

9 http://www.etvi.villena.es 
acción de gobierno, implicando siempre a la población, a los sectores privados y a los poderes públicos locales. De este modo, la ciudadanía ha asumido de forma voluntaria un papel activo por medio de convocatorias abiertas y sectoriales, a las que han acudido personas y colectivos de todo tipo. El motivo de esta dinámica de trabajo ha sido avanzar en un doble proceso: la obtención de datos (talleres, entrevistas, jornadas) y la determinación de prioridades y necesidades (asambleas ciudadanas), generando, al mismo tiempo, una mayor implicación de los actores municipales en el desarrollo de la Estrategia y en el compromiso de la aplicación de sus resultados.

En ese marco de actuación se plantea la realización de una serie de encuentros abiertos a la ciudadanía mediante el formato de asambleas, con el objetivo de valorar la posibilidad de que el municipio se dotase de una estructura de carácter consultivo del tipo Consejo Social de la Ciudad. En las diferentes sesiones mantenidas se ha reflexionado sobre su oportunidad, orientación y bases constitutivas, concluyéndose la necesidad de articular un proceso de trabajo protagonizado por vecinos y vecinas de Villena y facilitado por la Estrategia, como fórmula más conveniente para conseguir articular una dinámica de acción ciudadana que asegure dos premisas fundamentales: a) configurar una estructura participativa a la mediad de Villena; y b) establecer una estructura participativa con amplia base social ${ }^{10}$.

Previo al inicio del proceso de definición de las bases que deben orientar la constitución de la estructura participativa, se reflexionó acerca de cuáles deberían ser sus principales identificadores, para asegurar realmente su carácter participativo y obtener el mejor resultado posible de la dinámica de trabajo abierta, con la que se ha pretendido asimismo animar el contexto socioeconómico municipal. De este modo, se formularon hasta diez recomendaciones generales, que se han seguido a lo largo de los dos años y medio que ha abarcado la labor realizada antes de la constitución del nuevo órgano en 2015. Son las siguientes:

- Definir el instrumento más apropiado para el municipio considerando sus particularidades.

- Aprovechar el proceso de definición para motivar la participación social y educar en su expresión.

- Concebir un instrumento operativo y que sea un revulsivo en la escala municipal.

- Desarrollar un instrumento versátil que sirva para todo(s) o para muchas cosas.

- Configurar un instrumento desde el consenso y hacerlo con todos y todas desde el principio.

10 El autor de la ponencia participó en el equipo de trabajo de la ETV+i, ocupándose específicamente de dinamizar el proceso de conformación del Foro Económico y Social. 
- Adecuar el proceso a los tiempos de las personas, de las entidades y del municipio.

- Conseguir la participación e intermediación de personas y entidades independientes.

- Conferir la máxima transparencia al proceso de definición e implementación de la estructura participativa.

- Registrar y difundir de modo adecuado todo el proceso y dotarlo de organización y rigurosidad.

- Trabajar en paralelo en la racionalización de las estructuras participativas existentes en el municipio.

Se concluyó asimismo que el contexto de trabajo propiciado por la ETV+i podría ser una oportunidad y adecuado marco de actuación para el avance del proceso de configuración de la estructura participativa, por lo que se acordó que aquélla asumiera un papel preferente en cuanto a su impulso y acompañamiento inicial, por lo menos hasta que la ciudadanía y las Instituciones municipales fuesen ocupando el lugar que les correspondiera en el mismo a partir de su progresiva involucración. De este modo, se diseñó un itinerario de encuentros durante la segunda mitad del año 2012 y primeros meses de 2013, abiertos a la participación ciudadana y dinámicos en cuanto a su desarrollo, para enganchar a las personas que intervenían en las distintas asambleas convocadas.

Durante los primeros encuentros se han ido formulando de manera colaborativa las bases sobre las que asentar la labor más específica de configuración de la estructura participativa, resultado una dinámica de trabajo altamente satisfactoria y rica en cuanto a las conclusiones obtenidas, que han contribuido a guiar el proceso en las siguientes fases. En este sentido, ha sido fundamental partir de la definición de elementos clave o referentes, que en cualquier situación orienten de modo adecuado las decisiones de los actores que se relacionan y protagonizan el proceso, con independencia del momento en que se han incorporado al mismo. Se pretendió primero responder a una serie de cuestiones estratégicas vinculadas con la estructura participativa que se deseaba conformar:

- ¿A qué necesidad responde? Responde a una cierta insatisfacción general que anima a cambiar las formas de participar en el proceso de desarrollo municipal, mediante el impulso de estrategias colaborativas e integradoras.

- ¿Qué ventajas reportará? Reportará un cauce de diálogo y acción conjunta que permitirá construir un municipio que responda a un modelo compartido.

- ¿Qué dificultades encontraremos? Habrá que salvar la desconfianza, superar los intereses particulares y gestionar la frustración e impaciencia que provocan las experiencias previas. 
- ¿Qué hemos aprendido de los antecedentes? Hemos aprendido que disponemos de capital humano e iniciativa para construir nuevos procesos, en los que el liderazgo compartido y la constancia que asegure la continuidad son imprescindibles.

- ¿Quién y qué entidad no debe faltar? Debe participar toda aquella persona y colectivo que desee aportar su punto de vista y su colaboración para mejorar de forma constructiva la realidad municipal haciendo sostenible el proceso.

- ¿Cómo iniciamos el proceso? Dialogando y consensuando objetivos, procedimiento y calendario de trabajo que haga avanzar un proceso ya iniciado en el que deben seguir integrándose actores todavía ausentes.

Y precisamente poniendo en práctica las pautas fijadas en esta última respuesta se pretendió profundizar en el proceso ya iniciado. En primer lugar, avanzando en lo relativo a los valores y claves de funcionamiento de la estructura participativa, de modo que, a través de la aportación colectiva, se obtuvieron múltiples puntos de encuentro acerca de los fundamentos que deben guiar su constitución y desarrollo posterior ${ }^{11}$. Tras esto y en segundo lugar, se formuló una pregunta directa a las personas participantes, en relación con sus condiciones para apoyar el proceso de constitución. Se pretendió con ello, por una parte, hacer visibles los obstáculos que pudieran surgir durante el proceso, y por otra, compartir los requerimientos y las aspiraciones no verbalizadas pero fundamentales para avanzar en el proyecto colectivo ${ }^{12}$.

Las premisas definidas conjuntamente son las que asimismo deberían orientar la actividad de un Grupo de Trabajo, reducido y surgido de los encuentros asamblearios, que se tendría que encargar de elaborar una propuesta de estructura de carácter consultivo adaptada a las particularidades de Villena. Así se confiere el máximo protagonismo a la ciudadanía, que participa en las convocatorias abiertas y realizadas en un lugar central y conocido del

11 Estos valores y claves son los siguientes, organizados según orden alfabético, sin intención de destacar ninguno de ellos: actitud positiva, acuerdo, adaptación, coherencia, conciliación, confianza, confluencia, consenso, constancia, coordinación, creatividad, diálogo, dinamizadora, disposición al cambio, empatía, esperanza, generosidad, honestidad, ilusión, implicación, influencia, integración, involucración, legitimación, motivación, optimismo, perseverancia, perspectiva constructiva, relación, resultados, sentimiento de ciudad, trabajo intergeneracional, útil, visión a largo plazo, voluntad y voluntad de servicio.

12 Las cuestiones formuladas fueron las siguientes: ¿Apoyaría esta iniciativa? ¿Cuáles son sus condiciones? Siendo éste el resultado de la consulta: a) Formuladas en sentido positivo: Que... sea útil; sea ilusionante; sea respetada; pasen cosas y genere acción y resultados; tenga resultados no inmediatos pero no excesivamente dilatados en el tiempo; beneficie al conjunto de la ciudadanía de Villena; estemos todas y todos incluidos; sea sostenible o perdure en el tiempo y tenga garantía de continuidad; las propuestas sean sensatas; exista coherencia, constancia y compromiso a largo plazo; haya consenso político; tenga en cuenta lo más frágil; sea vinculante; los resultados y las conclusiones se apliquen sean cuales sean. b) Formuladas en sentido negativo: Que... no haya vetos; no sea un grupo de presión; no existan intereses personales, particulares o de grupo; no se paralice. 
municipio, facilitadas por el equipo de la ETV+i. El resultado de la labor de dicho Grupo sería presentado a las personas que vienen reuniéndose periódicamente de manera asamblearia, de modo que, se pueda conformar una propuesta definitiva que elevar al Pleno del Ayuntamiento para su aprobación definitiva. Las características y orientaciones para el más adecuado funcionamiento del Grupo de Trabajo se establecen antes de la designación de sus componentes ${ }^{13}$.

Y así, se eligió mediante convocatoria pública a doce ciudadanos/as como Grupo de Trabajo con carácter gestor, con el fin de que elaborara un documento de bases para la constitución del futuro Foro Económico y Social de Villena. El mandato de la Asamblea al Grupo de Trabajo fue el siguiente: "Elaborar un documento de base para la creación de una estructura del tipo Consejo Social de la Ciudad". Asimismo se enuncian una serie de premisas para garantizar la sostenibilidad y la calidad del proceso participativo que concluirá con la formulación de las directrices general del nuevo órgano participativo. Éstas tienen que ver con la actuación de las personas seleccionadas, que adquieren notable protagonismo a partir de entonces en relación con el impulso de las acciones conducentes a lograr el objetivo de dotar a Villena de un Foro Económico y Social, mediante una dinámica de trabajo que adquiere una dimensión cada vez más original por su desarrollo de abajo hacia arriba, situando a la ciudadanía en el centro del proceso.

Con el objetivo de centrar adecuadamente las tareas conducentes a la formulación de las bases de la estructura participativa, se define un esquema de temas o aspectos fundamentales que deben ser abordados y resueltos adaptándose a las particularidades de Villena. Se organizan en torno a cuatro grandes bloques: jurídico-administrativo, principios básicos, estructura y funcionamiento. Configuran lo que se vino a denominar Documento de Intenciones por parte del Grupo de Trabajo, elaborándose diversas versiones del mismo hasta su presentación definitiva a la Asamblea por dos veces, para ser ratificado por la misma después de la incorporación de distintas aportaciones fruto de los debates suscitados. En el proceso de elaboración de dicho documento se establecen

13 El Grupo de Trabajo debería responder a las siguientes características: constituido por un número de personas voluntarias, que oscila entre 8 y 12 componentes, manteniendo un equilibrio entre géneros e intentando que el grupo refleje la diversidad municipal; integrado por personas dialogantes y facilitadoras, con disponibilidad y capacidad de trabajo y comunicación; se reúne una vez al mes como mínimo mediante encuentros operativos manteniendo comunicación durante cada semana; debe tener preparado un primer borrador de propuesta de estructura consultiva a los seis meses del inicio de su actividad; presenta la labor realizada cada trimestre a la asamblea general durante el proceso de trabajo grupal; durante la primera sesión se organizarán de la forma más conveniente para cumplir sus objetivos, elaborando un plan de trabajo y concretando la manera más efectiva de repartir la tarea entre sus integrantes; debe llevar un registro de su actividad y actas de las sesiones celebradas, que se convocarán con orden del día; puede contar con la colaboración de personas para facilitar el proceso de trabajo, preferentemente vinculadas con la ETV+i. 
asimismo los centros de interés del Grupo de Trabajo, a modo de ámbitos de reflexión y debate que permitan enriquecer la propuesta final ${ }^{14}$.
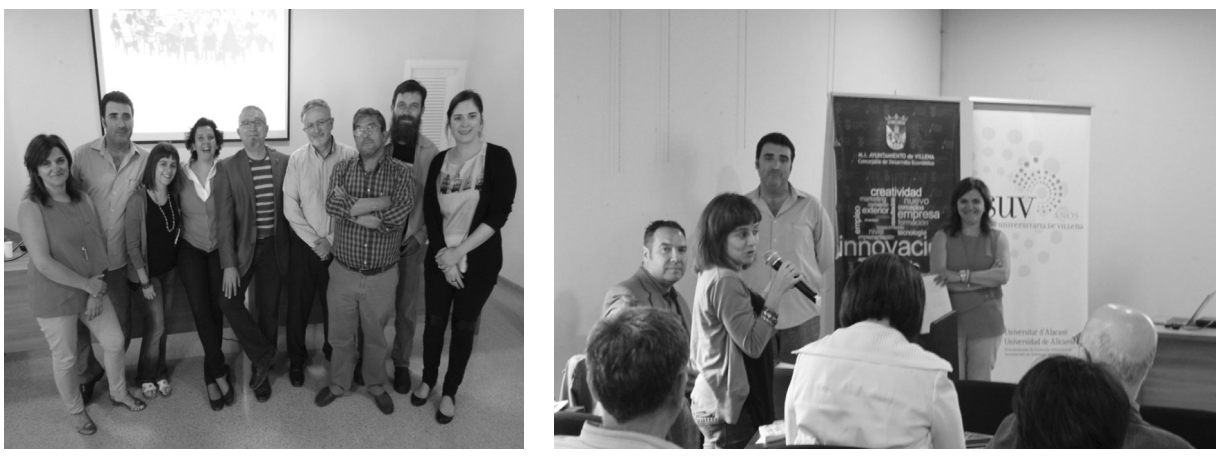

Foto 9 y 10. Desarrollo de la presente ponencia en la Sede Universitaria de Villena de la UA (La Tercia), junto con los miembros del grupo promotor del Foro Económico y Social en el marco del IX Coloquio de Desarrollo Local del GTDL-AGE.

Fuente: Salvador Martínez Puche

La Declaración de Intenciones se obtiene entonces a través del desarrollo de un proceso participativo que se ha alargado por espacio de un año y medio, en el que han podido intervenir los componentes del Grupo de Trabajo incorporando sus propuestas de mejora en sucesivas reuniones, enriquecidas asimismo con las sugerencias de los partidos políticos, sindicatos, Consejo de la Juventud y diversas asociaciones sectoriales de Villena. El objetivo general que se va conformando con el órgano propuesto es estimular a la ciudadanía, favoreciendo su implicación en el devenir del municipio, dirigido a generar aportaciones que mejoren la dinámica y calidad económica y social de la ciudad y su término municipal. Asume por lo tanto una función de dinamizador y conector de los actores locales.

Y es que, el Foro Económico y Social de Villena como facilitador de la participación se organiza con un fin eminentemente dinamizador, en paralelo a su objetivo carácter consultivo, impulsando la comunicación y la acción positiva de la ciudadanía que se implica de forma responsable y activa en reflexionar, debatir y aportar o proponer soluciones e iniciativas. Será la puesta en marcha

14 Estos centros de interés son los siguientes: revisión de experiencias anteriores y conocimiento de las causas que no permitieron su concreción y/o consolidación (obstáculos internos y externos); papel de la dimensión política y de la vinculación del órgano con la administración municipal; grado de autonomía -o independencia- que asegure su permanencia y sostenibilidad, operatividad y credibilidad; estructura con iniciativa propia que supere el mero carácter consultivo para ahondar en su vertiente propositiva; organización que permita dar cabida al conjunto de actores locales y refleje la pluralidad municipal; órgano próximo y dinámico que permita un flujo de ida y vuelta en relación con la ciudadanía y sus organizaciones. 
de éstas la que planteará necesidades, presupuestarias y de otro tipo, para proyectos que mejoren el proceso de desarrollo de la ciudad y su término municipal desde una perspectiva integral. De hecho, buena parte de las discusiones generadas durante la conformación de las bases de la estructura participativa, se relacionan con la posibilidad de que el Foro pueda desarrollar funciones más que consultivas.

En este sentido, el proceso de trabajo seguido por el grupo de ciudadanos/ as y sus facilitadores, ha estado caracterizado por la búsqueda de las mejores alternativas de cara a la definición de los fundamentos para la conformación de un órgano de participación a la medida de Villena, procurando siempre alcanzar consensos sobre los temas debatidos, de modo que se consiguiera una propuesta consistente y coherente desde las distintas visiones que se derivan de la propia diversidad del Grupo. La consulta a diferentes actores municipales y su presentación pública en asamblea, abriéndose a la participación de más vecinos y vecinas de Villena, concluye con la elaboración de un documento de principios que se eleva al Ayuntamiento para su conversión en una propuesta de Reglamento.

A partir de aquí, el Grupo de Trabajo se transforma en Grupo de Seguimiento de la nueva etapa, en la que el Ayuntamiento de Villena ha puesto los medios necesarios para la elaboración de un Proyecto de Reglamento del Foro Económico y Social, aprobado más tarde por unanimidad de su propio Pleno. Durante ese tiempo se ha seguido informando a la ciudadanía de Villena de los avances realizados y de los hitos del proceso, desplegando una intensa campaña de comunicación. Se puso fin así de manera satisfactoria a la conformación de la estructura participativa, abriéndose otra de mayor protagonismo de la entidad municipal con el acompañamiento del Grupo de Seguimiento, renovado en algunos de sus componentes con respecto a su configuración inicial.

Todo este inmenso trabajo colaborativo ha concluido con la constitución del Foro, siguiendo las pautas definidas inicialmente y refrendadas más tarde por el Pleno Municipal. Durante esta última fase se ha seguido haciendo énfasis en la comunicación de su existencia y posibilidades, evaluándose incluso el proceso que derivó en su concreción y apostando además por la adquisición de nuevo conocimiento y capacitación de sus componentes, como fórmula para su más adecuado desarrollo. Asimismo se ha invertido tiempo en perfilar protocolos de funcionamiento, con el fin de mejorar sus prestaciones, siempre propiciando el debate interno y el trabajo común, refiriéndose a las directrices acordadas al comienzo del recorrido conjunto.

Con la perspectiva de más de dos años de trabajo comunitario en un contexto no exento de dificultades, es posible extraer una serie de conclusiones que enfatizan el carácter original de la dinámica social suscitada en Villena. A modo sintético, se recogen a continuación: 
- Se trata de un proceso que se ha ido construyendo mediante la aportación colectiva, adquiriendo vitalidad y dinámica propia conforme va pasando el tiempo; pese a que ha sido facilitado por especialistas en trabajo comunitario, ha ido orientándose de manera particular y generando novedades en función de la participación ciudadana suscitada.

- Se ha desarrollado un proceso simultáneo de motivación y refuerzo de las posibilidades de participación social en la escala municipal (autoestima comunitaria), en el que la ciudadanía, las instituciones y las organizaciones de Villena han ido adquiriendo creciente protagonismo.

- Se ha superado el mero horizonte o reto particular de configurar una estructura participativa, evidenciando que la ciudadanía junto a la dimensión política y la suma de otros actores económicos y sociales, pueden aspirar a la construcción conjunta de procesos enormemente revitalizadores.

- Se han validado fórmulas e instrumentos de acción comunitaria que aseguran la sostenibilidad de los procesos sociales, como la construcción compartida y el trabajo colaborativo, el avance mediante el consenso, la transparencia y la horizontalidad que confieren similar protagonismo a todas las personas participantes.

- Se ha mantenido la confianza en el devenir de un proceso construido al ritmo que han marcado sus protagonistas, a partir de la generación inicial del ambiente propicio que ha permitido enfocarlo mediante el planteamiento y desarrollo de hitos-encuentros estimulantes con la idea de su cristalización a medio plazo.

- Se ha puesto de manifiesto el trascendente papel que puede desempeñar la Universidad como agente facilitador, dinamizador y mediador en procesos que avanzan fruto de la confluencia de múltiples actores sociales y económicos, aportando elementos conceptuales y metodológicos junto a una posición de equidistancia que propicia la conformación de un clima de confianza y respeto mutuo.

- Se ha contrastado la importancia de la implicación municipal 'sin reservas' en sus diferentes dimensiones, política y técnica, con el fin de afianzar el proceso también desde la Administración sin pretender apropiarse del mismo.

La experiencia de conformación de un órgano participativo de carácter económico y social en Villena, ha puesto de manifiesto la trascendencia de apostar por el desarrollo de estrategias de relación que se hilvanen con enfoque ascendente. También por la generación de estructuras fundamentadas en la confianza, surgidas de procesos de confluencia e intervención conjunta. La construcción de redes de trabajo, basadas en objetivos compartidos y en la cohesión que produce el hacer juntos, es sin duda esencial en este sentido. 
Redes que además favorezcan la autonomía y propicien descentralizar la acción a través de la constitución de grupos operativos. Y que permitan, asimismo, orientar la actuación de los actores hacia ámbitos que vayan centrando su interés preferente en cada momento. Esto influye tanto en la nueva organización que se pretende configurar como en la maduración individual de cada participante, al enriquecerse del aprendizaje que resulta del quehacer colectivo. Encuentro, diálogo, cohesión, planificación, liderazgo, entre otros, suponen elementos que se deben manejar bien para la mejor gestión de las redes y sus ramificaciones.

\section{Conclusiones}

Conocemos nuevos territorios y nos sigue sorprendiendo el débil protagonismo que ofrece la ciudadanía en el proceso de desarrollo compartido, pese a las múltiples recomendaciones y oportunidades existentes para enfrentar la mejora de la realidad social suscitando transformaciones que aprovechen el potencial de la creatividad comunitaria. La experiencia adquirida en décadas de proyección de los principios del desarrollo local parece que todavía no es suficiente, incluso mediante el refuerzo que ha supuesto el compromiso de organizaciones como la Unión Europea, puesto que, ante todo, estamos frente a obstáculos que tienen que ver con una cultura de hacer las cosas que sigue sin poner el acento lo necesario en el hacer juntos.

La Democracia Representativa tampoco lo pone fácil, dado que enfatiza el sentido pleno de la delegación y el protagonismo de la dimensión política; junto a una esfera técnico-profesional enredada en muchos casos en entornos enormemente burocratizados, se ensombrece a menudo la posibilidad de que la ciudadanía adquiera cada vez más relevancia en la definición del proyecto de territorio, y por lo tanto, en el buen gobierno y la optimización de los recursos propios. Allí donde se promueven procesos inclusivos con la pretensión de hacer y fortalecer el sentimiento de comunidad, mediante la participación de todos sus actores, se producen resultados realmente alentadores, validando las premisas que aporta el concepto y la metodología del desarrollo local. Sobre todo ocurre cuando la participación se convierte en un instrumento útil a los objetivos comunitarios y no en un fin en sí misma. De este modo y llegados a este punto, es posible formular una serie de $\operatorname{retos}^{15}$ en participación:

- Que la participación sirva a los fines del desarrollo, general y particular, devolviendo el protagonismo a las comunidades locales, con la aspiración de que sean capaces de formular proyectos en el sentido de estrategias de desarrollo de base comunitaria para cada territorio.

15 Formulados en parte con motivo del desarrollo del I Congreso Iberoamericano de Innovación Pública (NovaGob 2014), celebrado en la isla de Tenerife durante el mes de noviembre de 2014, en el marco del diálogo dedicado específicamente a reflexionar acerca de los retos en participación. 
- Que identifiquemos referencias comunes o colectivas o comunitarias, con el sentido de trascender de mi tema a nuestro tema, en el marco de procesos comunitarios inclusivos que alienten y garanticen la más amplia participación posible.

- Que encontremos fórmulas organizativas adecuadas en el marco local, partiendo de lo que ya existe y definiendo estrategias para articular de la mejor forma posible lo que se ha ido creado junto a las nuevas aportaciones: "construimos lo global desde lo local" (Marco Marchioni).

- Que podamos asegurar la durabilidad, la autenticidad y la sostenibilidad de los procesos participativos, favoreciendo su autonomía para que puedan alcanzar sin desvirtuarse los objetivos que han sido definidos revisándolos de manera permanente.

- Que ideemos estrategias para que la participación sea gratificante y sobre todo útil a la ciudadanía, generando resultados concretos en el plano colectivo y también recompensas personales particularmente en la esfera de lo intangible.

En definitiva, que la comunidad sea cada vez más protagonista de su propio destino en un contexto de creciente y más rica Democracia Participativa. Objetivo en el que se deben empeñar los actores de cualquier lugar de forma solidaria, buscando el diálogo con horizontalidad, representando cada uno su papel específico, que pueda confluir en el establecimiento de acuerdos para gestionar la realidad compartida. Enfocado todo como un proceso de permanente innovación social, en el que la participación suponga el mejor instrumento para formular estrategias coherentes de desarrollo que favorezcan el progreso colectivo en el contexto local con perspectiva general.

\section{BIBLIOGRAFÍA}

FALS BORDA, R. BRANDAO, CETRULO (1987): Investigación participativa. Instituto del Hombre, Ediciones de la Banda Oriental, Montevideo.

FONT, J., MONTERO, J.R. y TORCAL, M. (2006) (editores): Ciudadanos, asociaciones y participación en España, Centro de Investigaciones Sociológicas (CIS), Madrid, $430 \mathrm{p}$.

MARCHIONI, M. (1987): "Reflexiones en torno a la metodología de la intervención social en las nuevas condiciones hoy", Documentación Social, 69, 53-67.

MARCHIONI, M. (1989): Planificación social y organización de la comunidad. Alternativas avanzadas a la crisis, Madrid Popular, (4 $4^{a}$ edición, existen otras ediciones posteriores, la última en 1997).

MARCHIONI, M. (1994): La Utopía posible. La intervención comunitaria en las nuevas condiciones sociales, Benchomo, La Laguna (Tenerife). 
MARCHIONI, M. (2006): "Democracia participativa y crisis de la política. La experiencia de los planes comunitarios", Cuadernos de Trabajo Social, Vol. 19 , pp. 213-224.

MARTÍNEZ, A., MARTÍNEZ, S., PALACÍ, J., ZAPATA, V.M. Y EXPÓSITO, M. (2013): "La estrategia territorial de Villena+innovación (ETV+i). Un proceso experimental y aplicado de participación ciudadana", en Calvo, R. et alii (coords.) Ciudadanía empoderada: cultura y participación para el desarrollo local, ed. Germania, Alzira, 309 p.

MARTÍNEZ, A., et alii, (2014): "Estrategias territoriales participativas como instrumentos de diagnóstico y prospectiva, en el contexto europeo de los fondos de cohesión 2014-2020: el caso de Villena (Alicante)", Espacio, tiempo y forma, Serie VI · Geografía 6-7, UNED, Madrid, pp. 161-183.

ROMERO, J. (2005): "El gobierno del territorio en España. Balance de iniciativas de coordinación y cooperación territorial” Boletín de la A.G.E., nº39, Madrid, pp. 59-86.

ROMERO, J. y FARINÓS, J. (2011): "Redescubriendo la gobernanza más allá del buen gobierno. Democracia como base, desarrollo territorial como resultado", Boletín de la Asociación de Geógrafos Españoles (AGE), Murcia, no 56, pp. 295-319.

VILLASANTE, T.R. (2006): Desbordes creativos. Estilos y estrategias para la transformación social, La Catarata, Madrid.

ZAPATA HERNÁNDEZ, V.M. (Dir.) (2008): Guía práctica para el trabajo técnico en desarrollo rural, Extensión Universitaria, Universitat Jaume I, Castellón, 219 p. 